ARTICLE

\title{
Chemoenzymatic modular assembly of O-GalNAc glycans for functional glycomics
}

\author{
Shuaishuai Wang (10 1,10, Congcong Chen²,3,10, Madhusudhan Reddy Gadi1,10, Varma Saikam¹,10, Ding Liu1,

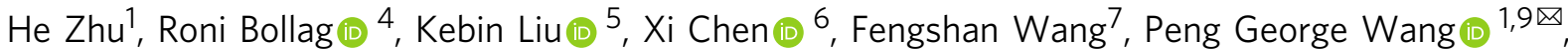 \\ Peixue Ling (10) $2,3,7 凶$, Wanyi Guan (i) ${ }^{8 凶}$ \& Lei Li (i) ${ }^{1 凶}$
}

O-GalNAc glycans (or mucin O-glycans) play pivotal roles in diverse biological and pathological processes, including tumor growth and progression. Structurally defined O-GalNAc glycans are essential for functional studies but synthetic challenges and their inherent structural diversity and complexity have limited access to these compounds. Herein, we report an efficient and robust chemoenzymatic modular assembly (CEMA) strategy to construct structurally diverse O-GalNAc glycans. The key to this strategy is the convergent assembly of O-GalNAc cores 1-4 and 6 from three chemical building blocks, followed by enzymatic diversification of the cores by 13 well-tailored enzyme modules. A total of 83 O-GalNAc glycans presenting various natural glycan epitopes are obtained and used to generate a unique synthetic mucin O-glycan microarray. Binding specificities of glycanbinding proteins (GBPs) including plant lectins and selected anti-glycan antibodies towards these O-GalNAc glycans are revealed by this microarray, promoting their applicability in functional O-glycomics. Serum samples from colorectal cancer patients and healthy controls are assayed using the array reveal higher bindings towards less common cores 3, 4, and 6 than abundant cores 1 and 2, providing insights into O-GalNAc glycan structure-activity relationships.

\footnotetext{
${ }^{1}$ Department of Chemistry, Georgia State University, Atlanta, GA 30303, USA. ${ }^{2}$ National Glycoengineering Research Center, Shandong Provincial Key Laboratory of Glycochemistry and Glycobiology, Shandong University, Qingdao 266237 Shandong, China. ${ }^{3}$ Shandong Academy of Pharmaceutical Science, Key Laboratory of Biopharmaceuticals, Engineering Laboratory of Polysaccharide Drugs, National-Local Joint Engineering Laboratory of Polysaccharide Drugs, Jinan 250101 Shandong, China. ${ }^{4}$ Georgia Cancer Center, Augusta University, Augusta, GA 30912, USA. ${ }^{5}$ Department of Biochemistry and Molecular Biology, Medical College of Georgia, Augusta, GA 30912, USA. ${ }^{6}$ Department of Chemistry, University of California, Davis, CA 95616, USA. ${ }^{7}$ Key Laboratory of Chemical Biology (Ministry of Education), Institute of Biochemical and Biotechnological Drug, School of Pharmaceutical Science, Shandong University, Jinan 250012 Shandong, China. ${ }^{8}$ College of Life Science, Hebei Normal University, Shijiazhuang 050024 Hebei, China. ${ }^{9}$ Present address: School of Medicine, Southern University of Science and Technology, Shenzhen 518055 Guangdong, China. ${ }^{10}$ These authors contributed equally: Shuaishuai Wang, Congcong

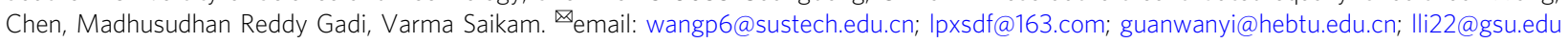


O -GalNAc glycans (also known as mucin O-glycans) represent a major component of the mammalian glycocalyx and are involved in various biological processes via glycan-protein interactions ${ }^{1}$. All O-GalNAc glycans share a common structural feature containing the monosaccharide $\mathrm{N}$ acetylgalactosamine (GalNAc) a-linked to the hydroxyl group of serine (Ser) or threonine (Thr) residues in proteins ${ }^{2}$. O-GalNAc glycosylation decorates over $80 \%$ of secretory and cell surface proteins $^{3}$. It confers many critical biological functions ranging from structural roles to immune responses and cell-cell interactions. For example, mucin is a major part of the mucosal barrier on gastrointestinal, respiratory, reproductive, and urinary tracts that protects humans from pathogens and aids clearance of microbes ${ }^{4}$. O-GalNAc glycans on mucins influence the configuration and exposure of peptide epitopes and the adhesive properties of glycoproteins ${ }^{1}$. On the other hand, pathogens could harness cell surface glycans as entry receptors to initiate infection $^{5}$, or produce surface glycans mimicking host O-GalNAc glycans to escape from the host immune surveillance ${ }^{6}$. Aberrant O-GalNAc glycosylation is correlated with tumor growth and metastasis by modulating tumor cell-matrix interactions ${ }^{7-9}$. As a result, some O-GalNAc glycans and glycopeptides have been used and explored as cancer biomarkers for clinical diagnosis and as antigenic targets for the development of therapeutic antibodies ${ }^{10,11}$

$\mathrm{O}-$ GalNAc glycans are structurally more diverse than $\mathrm{N}$ glycans. A single GalNAc residue $\alpha$-linked to Ser/Thr forms the Tn antigen (GalNAca-Ser/Thr), which is often a2-6 sialylated, providing the sialyl-Tn antigen (Siaa2-6GalNAca-Ser/Thr). Tn can also be extended to generate four major O-GalNAc cores (cores 1-4) and four rare cores (cores 5-8). As shown in Fig. 1, the attachment of a galactose $(\mathrm{Gal})$ residue to the Tn antigen via a $\beta 1-3$ linkage generates core 1 , also named $\mathrm{T}$ antigen. Core 2 is formed by adding an $\mathrm{N}$-acetylglucosamine (GlcNAc) residue to the GalNAc of core 1 via a $\beta 1-6$ linkage. Cores 1 and 2 glycans are the most common O-GalNAc structures and nearly ubiquitously expressed on mucins and other glycoproteins. Cores 3, 4, and 6 are $\beta$-GlcNAcylated on C3-hydroxyl $(\mathrm{C} 3-\mathrm{OH})$ and/or C6-OH of the initiating GalNAc, whereas cores 5,7 , and 8 contain $\alpha$-linked extensions ( $\alpha 1-3 \mathrm{GalNAc}, \alpha 1-6 \mathrm{GalNAc}$, and $\alpha 1-3 \mathrm{Gal}$, respectively) (Fig. 1a). Cores 3 and 4 are less common than cores 1 and 2, and are more restricted to glycoproteins in bronchial and gastrointestinal tissues ${ }^{1}$. Cores $5-8$ are linear structures with low occurrence and abundance. The diversity and complexity of

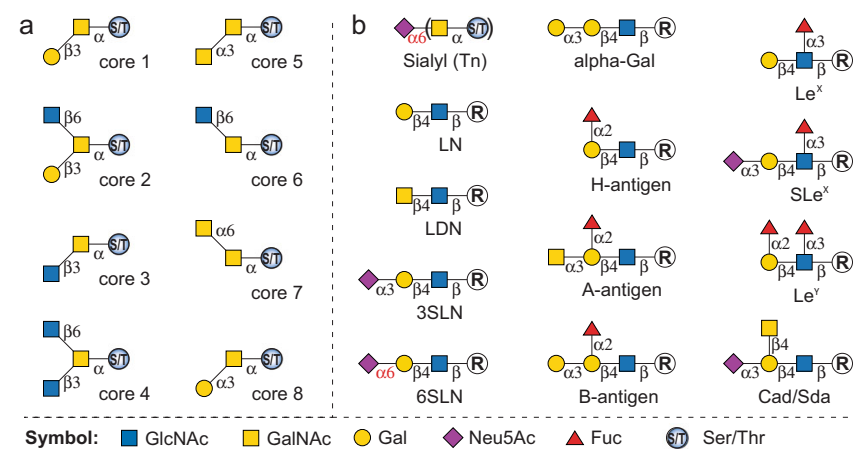

Fig. 1 Structures of O-GaINAc glycan cores and their common glycan epitope extensions. a O-GalNAc glycan cores 1-8. b Common glycan epitopes found on O-GalNAc glycans. LN, N-acetyllactosamine (LacNAc); LDN, N,N'-diacetyllactosamine (LacdiNAc); 3SLN, 3'-sialyl LacNAc; 6SLN, 6'-sialyl LacNAc; Le ${ }^{X}$, Lewis $X ;$ SLe $^{X}$, sialyl-Lewis $X ;$ Le $^{Y}$, Lewis $Y$. Abbreviations: Gal, galactose; Fuc, L-fucose; GlcNAc, N-acetylglucosamine; GalNAc, N-acetylgalactosamine; Neu5Ac, N-acetylneuraminic acid; Ser, serine; Thr, threonine.
O-GalNAc glycans stem from multiple cores and additional glycosylation presenting different epitopes. Sialylations are observed on all types of cores. Common epitopes identified on cores 1-4 and 6 include ABO blood group antigens (A-, B-, and $\mathrm{H}$-antigen), Lewis antigens (e.g., Lewis X, Lewis $\mathrm{Y}$, and sialylLewis X antigens), $N$-acetyllactosamine (LacNAc, LN), $N, N^{\prime}$ diacetyllactosamine (LDN), $3^{\prime}$-sialyl LacNAc (3SLN), $6^{\prime}$-sialyl LacNAc (6SLN), alpha-Gal, and Cad/Sd ${ }^{a}$ (Fig. 1b) ${ }^{1,10,12,13}$.

The access to structurally diverse O-GalNAc glycans in sufficient quantity and purity is essential to their structure-function relationship studies. In the last decade, shotgun glycomics ${ }^{14,15}$ oxidative release ${ }^{16}$, O-glycome beam search ${ }^{17}$, and preparative cellular O-glycome reporter/amplification ${ }^{18}$ have been developed to obtain O-GalNAc glycans from natural sources. However, these methods do not allow access to complex O-GalNAc glycans in sufficient quantity, especially for those with rare cores and low abundant glycoforms. Chemical and chemoenzymatic methods have thus been used to obtain a variety of sialylated O-GalNAc glycans ${ }^{19-23}$, including cores $1-4$ and 6 that contain poly-LacNAc motifs $^{24,25}$. In addition, solid-phase approaches have been developed for synthesizing O-GalNAc glycopeptides, but only those with simple core structures or their sialylated forms were normally obtained ${ }^{26-29}$. A strategy for the systematic preparation of O-GalNAc glycans with varied natural epitopes is still missing, and the lack of these structures had been a major barrier to functional O-glycomic studies.

Herein, we describe the implementation of a chemoenzymatic modular assembly (CEMA) strategy that deploys three synthetic building blocks and 13 enzyme modules in a precisely controlled manner for the rapid access of structurally diverse O-GalNAc glycans. The method yields a collection of $83 \mathrm{O}-\mathrm{GalNAc}$ glycans presenting important glycan epitopes on cores 1-4 and 6 . The attached Ser or Thr enables the easy attachment of O-glycans to glass surfaces for the fabrication of a unique O-GalNAc glycan microarray, which is a useful tool to investigate the glycanbinding specificity of glycan binding proteins (GBPs) and probe anti-mucin antibodies.

\section{Results and discussion}

The chemoenzymatic modular assembly (CEMA) strategy. The CEMA strategy includes (1) diversity-oriented and scalable chemical assembly of O-GalNAc glycan cores, and (2) highly efficient enzyme modules to glycosylate the cores with precise control on regio- and stereoselectivity. All O-GalNAc cores are branched from either the $\mathrm{C} 3-\mathrm{OH}$ or/and the $\mathrm{C} 6-\mathrm{OH}$ of the initiating $\mathrm{Gal}-$ NAc (Fig. 1a). Thus, a suitable Tn antigen selectively protected at $\mathrm{C} 3 / 6-\mathrm{OH}$ to allow late-stage selective deprotection is the key for assembling the cores. To access cores $1-4$ and 6 (1-5, Fig. $2 \mathrm{a})$, we designed three synthetic building blocks, including a versatile protected glycosyl amino acid 7 with $\mathrm{C} 3-\mathrm{OH}$ protected by an acetyl (Ac) group and $\mathrm{C} 4 / 6-\mathrm{OH}$ masked by a benzylidene acetal, and two monosaccharide Schmidt donors $\mathbf{8}^{30}$ and $\mathbf{9}^{31}$. The Fmoc group is introduced to the Ser residue of 7 to facilitate reaction monitoring and product purification ${ }^{32}$. The Ac group on 7 would be selectively removed under mild basic conditions. Subsequent branching at the free $\mathrm{C} 3-\mathrm{OH}$ by glycosylation with 8 and 9 would produce core 1 and 3 , respectively. In addition, deprotection of benzylidene acetal in the resulting disaccharides to expose $\mathrm{C} 4-\mathrm{OH}$ and $\mathrm{C} 6-\mathrm{OH}$ followed by regioselective glycosylation at the more active C6-OH with $\mathrm{N}$-Troc-protected module 9 will yield cores 2 and 4 , respectively. On the other hand, core 6 could be obtained by selective deprotection of $\mathrm{C6}-\mathrm{OH}$ of 7 followed by the regioselective glycosylation with 9 .

Stereoselective synthesis of Tn antigen with an $\alpha$-linked GalNAc remains a major challenging step for mucin O-glycan synthesis ${ }^{33,34}$. 


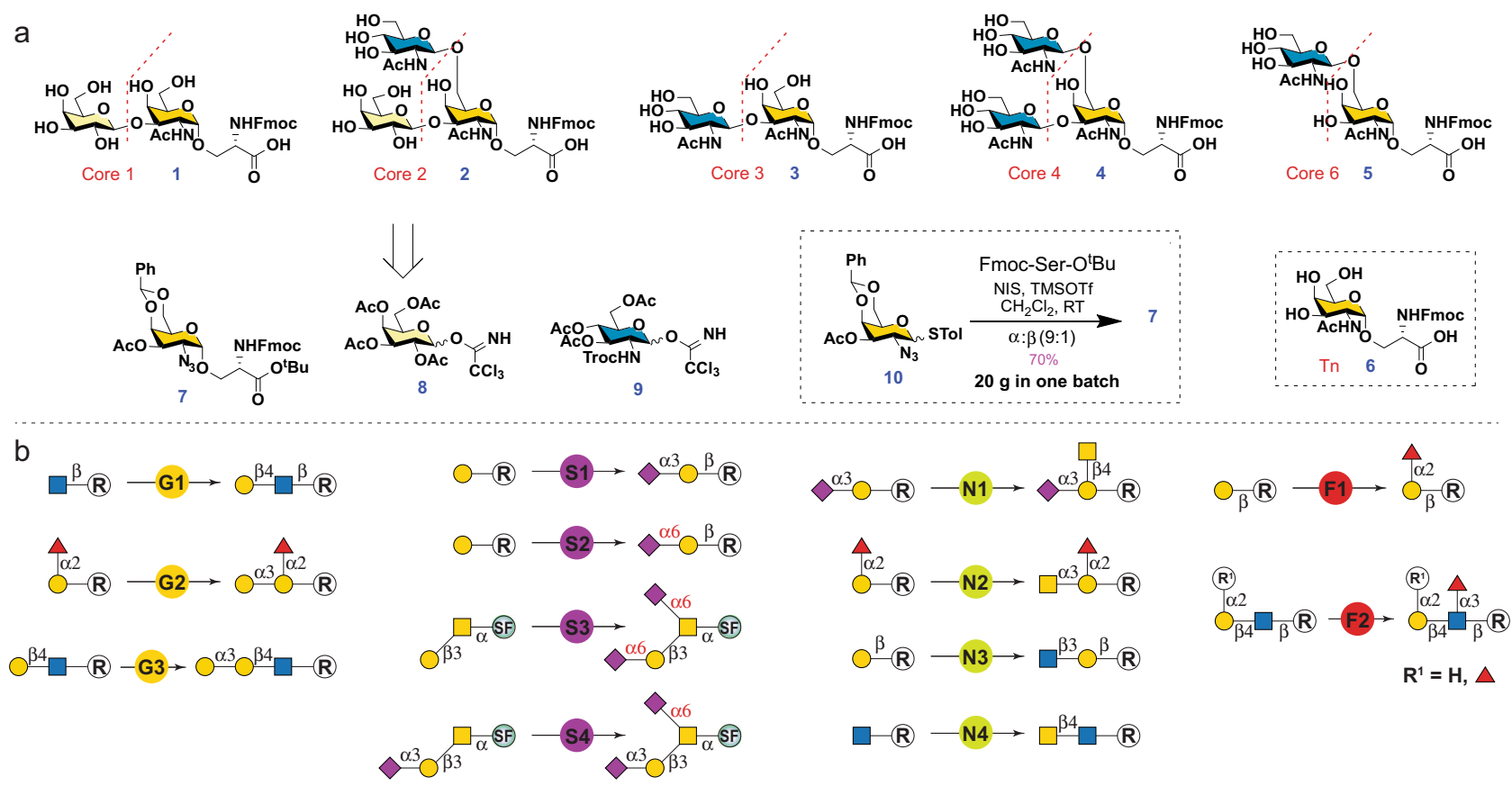

Fig. 2 Retrosynthetic analysis of O-GaINAc glycans. a Retrosynthetic analysis of O-GalNAc cores with three synthetic building blocks. b Retrosynthetic analysis of O-GalNAc epitopes with 13 enzyme modules. Enzyme modules: $\mathrm{G}$ stands for galactosylation, $\mathrm{S}$ stands for sialylation, $\mathrm{N}$ stands for $\mathrm{N}$-acetylhexosaminylation, F stands for fucosylation. G1: $\beta 1-4$ galactosylation with Neisseria meningitidis $\beta 1-4$ galactosyltransferase (NmLgtB) ${ }^{47}$ and donor uridine 5'-diphosphogalactose (UDP-Gal); G2: $\alpha 1-3$ galactosylation with human GTB and UDP-Gal; G3: $\alpha 1-3$ galactosylation with bovine $\alpha 1-3$ GalT (B $\alpha 3$ GalT) 48 and UDP-Gal; S1: $\alpha 2-3$ sialylation with Pasteurella multocida $\alpha 2-3$ sialyltransferase 1 mutant M144D (PmST1-M144D) 52 , N. meningitidis CMP-sialic acid synthetase (NmCSS) ${ }^{69}$, cytidine 5'-triphosphate (CTP), and N-acetylneuraminic acid (Neu5Ac); S2: $\alpha 2-6$ sialylation with PmST1-P34H/ M144L 49 , NmCSS, CTP, and Neu5Ac; S3: $\alpha 2-6$ sialylation with Photobacterium damselae $\alpha 2-6$ sialyltransferase (Pd2, 6ST) 50, NmCSS, CTP, and Neu5Ac; S4: $\alpha 2-6$ sialylation with human ST6GalNAc-IV, NmCSS, CTP, and Neu5Ac; N1: $\beta 1-4 \mathrm{~N}$-acetylgalactosaminylation with Campylobacter jejuni $\beta 1-4$ $\mathrm{N}$-acetylgalatosaminyltransferase (CjCgtA) ${ }^{70}$ and uridine $5^{\prime}$-diphosphate- $N$-acetylgalactosamine (UDP-GalNAc); N2: $\alpha 1-3 \mathrm{~N}$-acetylgalactosaminylation with Helicobacter mustelae $\alpha 1-3 \mathrm{~N}$-acetyl-galactosaminyltransferase (HmBgtA) ${ }^{56}$ and UDP-GalNAc; N3: $\beta 1-3 \mathrm{~N}$-acetylglucosaminylation with Helicobacter pylori $\beta 1-3 \mathrm{~N}$-acetylglucosaminyltransferase (HpLgtA) ${ }^{71}$ and uridine $5^{\prime}$-diphosphate- $N$-acetylglucosamine (UDP-GIcNAc); N4: $\beta 1-4$ Nacetylgalactosaminylation with b4GalT-Y289L/C342T (b4GalTm) 72 and UDP-GalNAc; F1: $\alpha 1-2$ fucosylation with $H$. mustelae $\alpha 1-2$ fucosyltransferase $(\mathrm{Hm} 2 \mathrm{FT})^{55}$ and guanosine 5'-diphospho-L-fucose (GDP-Fuc); F2: $\alpha 1-3$ fucosylation with H. pylori $\alpha 1-3$ fucosyltransferase C-terminal 66 amino acid truncation $(\mathrm{Hp} 3 \mathrm{FT})^{73}$ and GDP-Fuc. SF, Fmoc protected Ser.

High $\alpha$-selectivity is usually achieved by using a glycosyl donor with a non-participating group at $\mathrm{C} 2$, such as an azide group ${ }^{35}$. Extensive efforts have also been made on evaluating structural modification of sugar donors with various protective groups ${ }^{36-39}$, leaving groups ${ }^{40}$, and reaction conditions ${ }^{41,42}$. For the purpose of preparing structurally diverse O-GalNAc glycans, a simple, efficient, and diversity-oriented route encompassing a fluorescent core (e.g., Fmoc) would be more advantageous ${ }^{32}$. A stable thio glycosyl donor $\mathbf{1 0}^{43}$ was thus selected and obtained by efficient synthetic methods ${ }^{44}$. Ser with a Fmoc-protected amino group and a tertbutyl ester protected carboxyl group (Fmoc-Ser-O $\mathrm{O}^{t} \mathrm{Bu}$ ) was selected as the glycosyl acceptor ${ }^{45}$. Glycosylation of the Fmoc-Ser-O ${ }^{t} \mathrm{Bu}$ with the glycosyl donor $\mathbf{1 0}$ at room temperature promoted by NIS and TMSOTf produced $7^{41,46}$ with a satisfying yield of $70 \%$ and a good stereoselectivity of 9:1 ( $\alpha: \beta$ ) (Fig. 2a). Importantly, the presence of benzylidene acetal allowed easy separation of the anomers on TLC $\left(\mathrm{R}_{\mathrm{f}}\right.$ : $\alpha-0.7, \beta-0.4,50 \%$ ethyl acetate/hexanes) and efficient purification by silica gel flash column chromatography. The synthesis was easily scaled up to obtain 20 grams of 7 in one batch.

While chemical approaches are powerful in synthesizing simple cores for up to gram scales, enzyme-catalyzed reactions are advantageous in preparing large complex glycans owing to their unique regio- and stereoselectivity. To assemble common epitopes (Fig. 1b) on O-GalNAc cores 1-4 and 6, a total of 13 enzyme modules were employed (Fig. 2b), including three galactosylation modules (G1-G3), four sialylation modules (S1-S4), four
$\mathrm{N}$-acetylhexosaminylation modules (N1-N4), and two fucosylation modules (F1 and F2). Enzymes used in these modules are well characterized and robust glycosyltransferases (GTs). They are specific to generate only the desired epitopes. For example, Neisseria meningitidis $\beta 1-4$ galactosyltransferase $(\mathrm{NmLgtB})$ is used for $\beta 1-4$ galactosylation (module G1) ${ }^{47}$. Human GTB (module G2) and bovine $\alpha 1-3$ GalT $(\mathrm{Ba} 3 \mathrm{GalT})^{48}$ (module G3) are employed for a1-3 galactosylation to generate $\mathrm{B}$-antigen and alpha-Gal respectively, according to their acceptor specificities. For a2-6 sialylation, three enzyme modules are proposed: Pasteurella multocida a2-3 sialyltransferase 1 mutant P34H/ M144L (PmST1-P34H/M144L) that is highly selective for sialylating non-reducing terminal Gal residues $(\mathrm{S} 2)^{49}$, Photobacterium damselae a2-6 sialyltransferase $(\mathrm{Pd} 2,6 \mathrm{ST})$ that is highly active and recognizes all terminal and internal Gal and GalNAc $(\mathrm{S} 3)^{50}$, and human ST6GalNAc-IV that only recognizes the initiating GalNAc residue $(\mathrm{S} 4)^{51}$. All enzyme modules are welltailored for specific acceptors according to their substrate specificities to avoid side reactions and achieve precise control for the synthesis of desired glycans.

Chemical modular assembly of O-GalNAc cores 1-4 and 6. Module 7 serves as an advanced intermediate that can be extended properly at C3 or/and C6 positions to obtain all OGalNAc core structures (Fig. 3). To assemble core 1, the O-Ac 


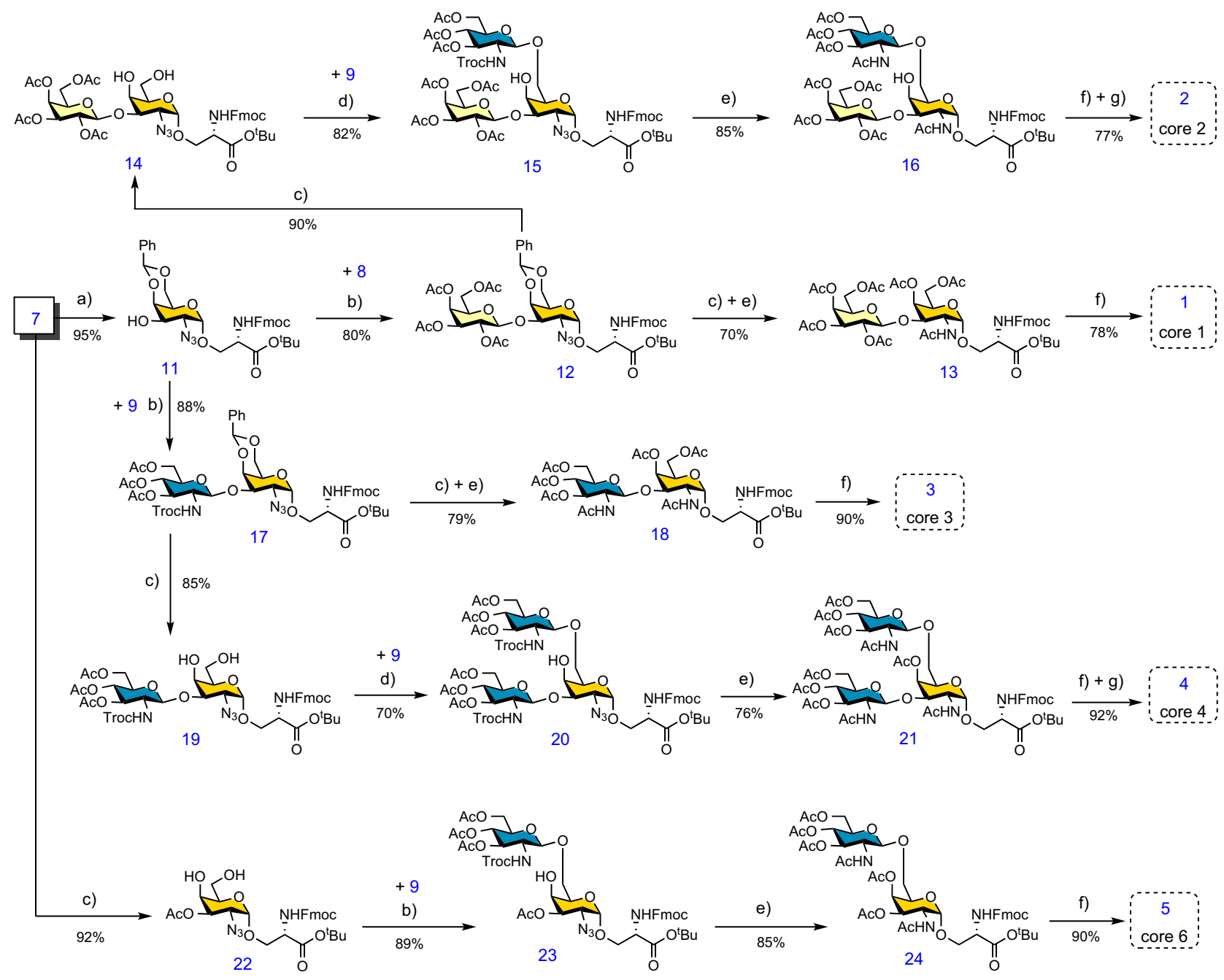

Fig. 3 Chemical modular assembly of O-GalNAc cores 1-4 and 6 structures. Reagents and conditions: a NaOMe, $\mathrm{MeOH} \mathbf{b} \mathrm{TMSOTf}_{1} \mathrm{CH}_{2} \mathrm{Cl}_{2},-78^{\circ} \mathrm{C}$. c $p$-toluenesulfonic acid, $\mathrm{MeOH}$. d TMSOTf, $\mathrm{CH}_{2} \mathrm{Cl}_{2},-40{ }^{\circ} \mathrm{C}$. e i. $\mathrm{Zn}, \mathrm{AcOH}_{2} \mathrm{CH}_{2} \mathrm{Cl}_{2} ;$ ii. $\mathrm{Py}, \mathrm{Ac}_{2} \mathrm{O}$. f i. TFA, $\mathrm{CH}_{2} \mathrm{Cl}_{2} ;$ ii. $\mathrm{NaOMe}, \mathrm{MeOH} \mathbf{g}$ FmocOSu, NaHCO .

group at $\mathrm{C} 3-\mathrm{OH}$ was deprotected under basic conditions to yield compound 11. The Schmidt donor module $\mathbf{8}$ was then used to glycosylate 11 in the presence of catalytic TMSOTf to provide the $\beta 1$-3-linked disaccharide 12. Subsequent deprotection of benzylidene acetal, azide reduction, and acetylation without any intermediate purification produced the protected core 1 (13). Finally, tert-butyl and Ac groups were removed under strong acidic and mild basic conditions successively to obtain Fmocprotected core 1 (1). It is worth noting to avoid the loss of Fmoc, the $\mathrm{pH}$ of the solution should not exceed 8.5 during the final Ac ester deprotection step. To synthesize core 2, the benzylidene acetal on 12 was removed to obtain diol 14. Regioselective installation of $N$-Troc protected module $9^{31}$ to the C6-OH of 14 using TMSOTf as a promoter resulted in the $\beta$-linked trisaccharide 15 exclusively. Successive deprotection of the $N$-Troc group, reduction of azide, and $\mathrm{N}$-acetylation resulted in the protected core 2 trisaccharide 16. Final deprotection of the tertbutyl and Ac esters produced core 2 (2). However, despite maintaining the $\mathrm{pH}$ of the reaction below 8.5 during Zemplén deprotection, nearly 50\% of Fmoc was lost. Fmoc was therefore quantitavely reintroduced under basic conditions using Fmoc $\mathrm{N}$-hydroxysuccinimide ester before product purification ${ }^{32}$. Core 2 (2) was obtained with a yield of $77 \%$ over the last three steps.

Cores 3, 4, and 6 are GlcNAc extended structures of Tn antigen at $\mathrm{C} 3$ and/or C6 positions, respectively. As illustrated in Fig. 3, to obtain core 3, glycosyl acceptor 11 was glycosylated with 9 in the presence of catalytic TMSOTf to obtain exclusively $\beta$-linked disaccharide 17. Subsequent deprotection of benzylidene acetal followed by one-pot $\mathrm{Zn}$-mediated reduction of azide and deprotection of $\mathrm{N}$-Troc formed a disaccharide with a free amino group at C-2, which was acetylated without purification to obtain 18. The ${ }^{t} \mathrm{Bu}$ and $\mathrm{Ac}$ esters were then deprotected to obtain core 3 (3). On the other hand, diol 19 obtained by benzylidene deprotection of $\mathbf{1 7}$ was glycosylated with $\mathbf{9}$ at the C6 position under Lewis acid conditions to provide $\mathbf{2 0}$ in a yield of $70 \%$. The protected derivative $\mathbf{2 0}$ was converted to the peracetylated compound 21 which was deprotected followed by the reintroduction of Fmoc to form core 4 (4) similar to that described above for the synthesis of core 2 . To access core 6 , benzylidene acetal was firstly removed to obtain $\mathbf{2 2}$ followed by regioselective glycosylation with 9 at C6, which provided $\beta$-linked disaccharide 23 exclusively. Subsequent $N$-Troc deprotection, azide reduction, and peracetylation yielded compound $\mathbf{2 4}$, which was converted to core 6 (5) by tert-butyl and Ac ester deprotection. The overall yields of cores 1-4 and 6 starting from module 7 are decent, ranging from $35 \%$ to $63 \%$. Lastly, the Tn antigen (6) was converted from the common intermediate $\mathbf{1 0}$ by azide reduction and global deprotection using contemporary chemical routes as described for the synthesis of core 1 (Supplementary Information). 
Enzymatic modular assembly of O-GalNAc glycans. With chemically synthesized Tn antigen, cores 1-4, and core 6 in hand, structurally diverse O-GalNAc glycans were prepared using 13 enzyme modules (Fig. 2b) in a well-designed sequential manner. Specifically, the Ser-linked STn antigen (25) was prepared from 6 by enzyme module S3. Briefly, Tn antigen (6) was incubated in $100 \mathrm{mM}$ Tris- $\mathrm{HCl}$ ( $\mathrm{pH} 8.0$ ) with the highly active Pd2,6ST, $N$. meningitidis CMP-sialic acid synthetase (NmCSS), cytidine 5'triphosphate (CTP), $N$-acetylneuraminic acid (Neu5Ac), and $\mathrm{MgCl}_{2}$. NmCSS enabled in situ generation of the sialyltransferase sugar nucleotide donor CMP-Neu5Ac. The reaction was carried out at $37^{\circ} \mathrm{C}$ for $3 \mathrm{~h}$ and stopped by boiling for $5 \mathrm{~min}$. After brief centrifugation, the supernatant was concentrated and purified by reverse-phase (RP)-HPLC (Supplementary Information) to afford the STn antigen (25). The bulky hydrophobic UV-detectable fluorescent Fmoc group facilitates real-time monitoring of reaction processes and makes the product separation by RP-HPLC easier ${ }^{32}$.

Core $1 \mathrm{O}$-GalNAc glycans are commonly found on mucins and other glycoproteins. Most core 1 structures are sialylated. As depicted in Fig. 4, enzymatic modular assembly starting from $\mathbf{1}$ yielded 19 extended core 1 structures, including 9 sialylated ones. Mono-sialylated glycans 26 and 27 were prepared by enzyme modules S2 and S1, respectively. As mentioned above, PmST1$\mathrm{P} 34 \mathrm{H} / \mathrm{M} 144 \mathrm{~L}$ in module $\mathrm{S} 2$ is an engineered regioselective $\alpha 2-6$ sialyltransferase ${ }^{49}$, whereas PmST1-M144D in S1 is a regioselective a2-3 sialyltransferase ${ }^{52}$. Both enzymes are highly selective for sialylating non-reducing end Gal residues. Core 1 with two a2-6linked sialic acids (28) was prepared by using module S3, which contains Pd2,6ST, a highly active a2-6 sialyltransferase with substrate promiscuity that recognizes both terminal and internal $\mathrm{Gal} / \mathrm{GalNAc}$ residues. Given that $\mathrm{Pd} 2,6 \mathrm{ST}$ could sialylate Gal even in the presence of $\alpha 2-3$ sialyation $^{53}$, the synthesis of disialylated 29 Neu5Aca2-3Galß1-3(Neu5Aca2-6)GalNAca-FmocSer was achieved via another $\alpha 2-6$ sialyation module $S 4$, in which human ST6GalNAc-IV catalyzed regioselective a2-6 sialylation of the initiating GalNAc ${ }^{51}$. O-GalNAc glycans 30 and 31 that presenting the $\mathrm{Cad} / \mathrm{Sd}^{\mathrm{a}}$ antigen were assembled by module $\mathrm{N} 1$ from 27 and 29, respectively. Enzyme module N1 contained $C$. jejuni $\beta 1-4-N$-acetylgalactosaminyltransferase (CjCgtA), which catalyzed the transfer of GalNAc onto the Gal residue of the Siaa2-3Gal motif ${ }^{54}$.

Additionally, core 1 with blood group H-antigen (32) was prepared by incubating 1 with enzyme module F1 that contained H. mustelae a1,2-fucosyltransferase $(\mathrm{Hm} 2 \mathrm{FT})^{55}$ and the sugar nucleotide GDP-Fuc. We found that Hm2FT not only recognized lactose $^{55}$, but also tolerated LacNAc and Gal $\beta 1-3$ GalNAc. It was used to synthesize O-GalNAc glycans bearing Type II and III $\mathrm{H}$-antigens (e.g., 43 and 32) in excellent yields. Glycans with

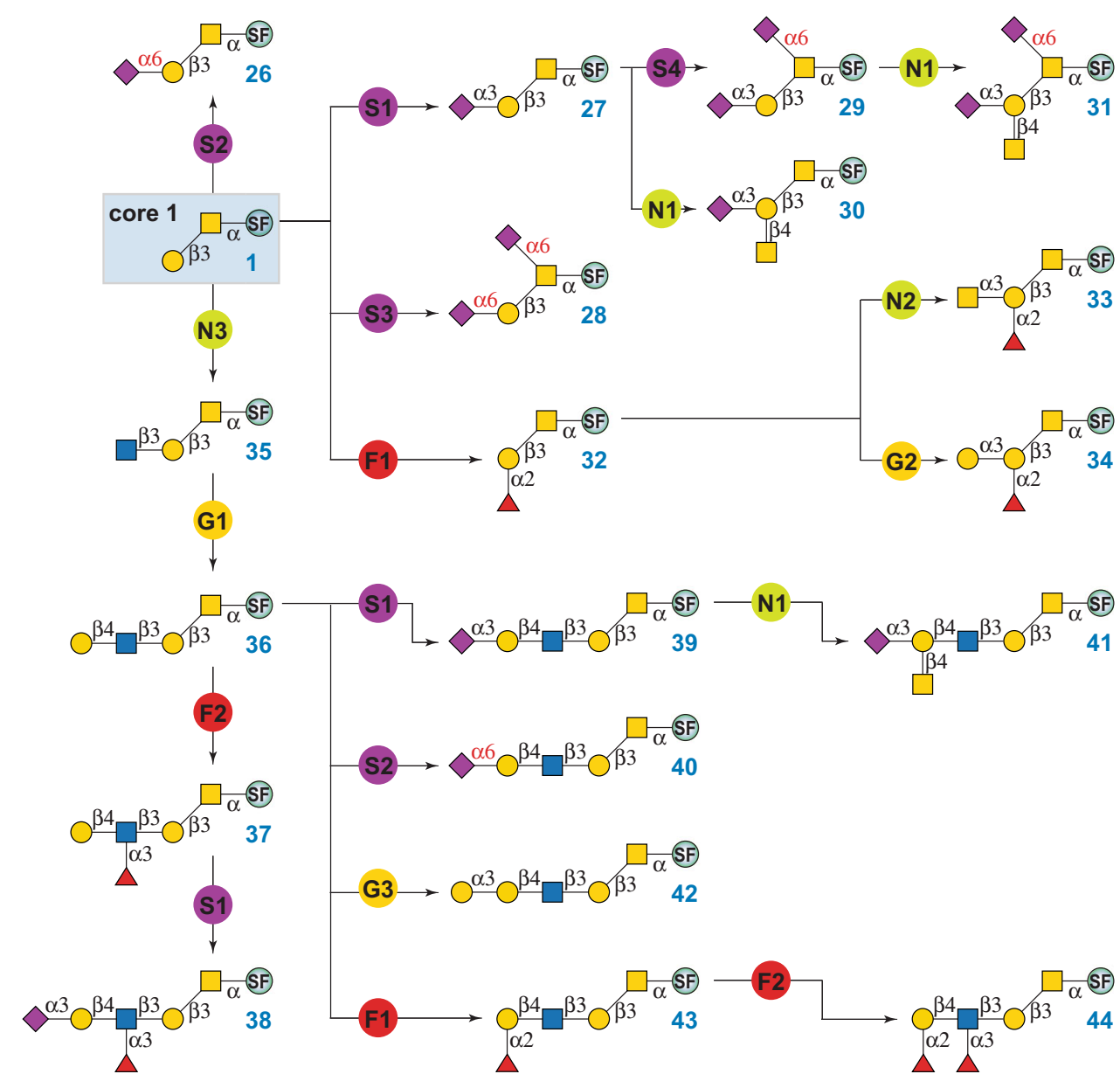

Fig. 4 Enzymatic modular synthesis of core 1 0-GalNAc glycans. G1: $\beta 1$-4 galactosylation with NmLgtB and UDP-Gal; G2: $\alpha 1-3$ galactosylation with human GTB and UDP-Gal; G3: $\alpha 1-3$ galactosylation with B $\alpha 3$ GalT and UDP-Gal; S1: $\alpha 2-3$ sialylation with PmST1-M144D, NmCSS, CTP, and Neu5Ac; S2: $\alpha 2-6$ sialylation with PmST1-P34H/M144L, NmCSS, CTP, and Neu5Ac; S3: $\alpha 2-6$ sialylation with Pd2,6ST, NmCSS, CTP, and Neu5Ac; S4: $\alpha 2-6$ sialylation with human ST6GaINAc-IV, NmCSS, CTP, and Neu5Ac; N1: $\beta 1-4$ N-acetylgalactosaminylation with CjCgtA and UDP-GalNAc; N2: $\alpha 1-3$ Nacetylgalactosaminylation with HmBgtA and UDP-GalNAc; N3: $\beta 1-3 \mathrm{~N}$-acetylglucosaminylation with HpLgtA and UDP-GIcNAc; F1: $\alpha 1-2$ fucosylation with Hm2FT and GDP-Fuc; F2: $\alpha 1-3$ fucosylation with Hp3FT and GDP-Fuc. SF, Fmoc protected Ser. 
blood group A- or B-antigens were subsequently prepared by $H$. mustelae a1,3- $N$-acetylgalactosamanyltransferase (HmBgtA) (module N2) and human GTB (module G2), respectively. Both enzymes have broad acceptor specificities towards all five subtypes of $\mathrm{H}$-antigens ${ }^{56,57}$.

Similarly, extended core 1 structures presenting LacNAc (36), $\mathrm{Le}^{\mathrm{X}}$ (37), SLe ${ }^{\mathrm{X}}(38), 3 \mathrm{SLN}$ (39), 6SLN (40), Cad/Sd ${ }^{\mathrm{a}}$ (41), alpha-Gal (42), $\mathrm{H}$-antigen (43), and $\mathrm{Le}^{\mathrm{Y}}$ (44) epitopes were prepared by the stepwise enzymatic modular assembly (Fig. 4). The assembly route was designed according to the acceptor specificity of each glycosyltransferase to avoid undesired side reactions. To further eliminate undesired side products, glycan products were purified by RP-HPLC to homogeneity ( $>98 \%$ ) before subjected to the next step. All purified glycans were characterized by analytical HPLC to confirm purity, and NMR and mass spectrometry to confirm structures (Supplementary Information). Please note that instead of multi-enzyme systems as that for in situ generation of CMPNeu5Ac, partially purified (P2-chromatography to $>70 \%$ ) sugar donors UPD-Gal, UDP-GalNAc, UDP-GlcNAc, and GDP-Fuc were used in modules G1-G3, N1-N4, and F1-F2, to reduce incubation times, increase yields, and simplify the HPLC purification process.

Core $2 \mathrm{O}$-glycans are branched structures where the $\beta 1-3 \mathrm{Gal}$ branch is commonly sialylated or attached with the $\mathrm{Cad} / \mathrm{Sd}^{\mathrm{a}}$ antigen and the $\beta 1-6$ GlcNAc branch presents varied epitopes ${ }^{12}$. Starting from 2 , a total of 21 core 2 structures were prepared via a sequential modular assembly of the $\beta 1-3 \mathrm{Gal}$ branch and then the $\beta 1-6 \mathrm{GlcNAc}$ branch (Fig. 5). For example, to prepare core 2 glycans with $\mathrm{Cad} / \mathrm{Sd}^{\mathrm{a}}$ antigen at the $\beta 1-3 \mathrm{Gal}$ branch (compounds 46-49), a2-3 sialylation of core 2 (2) was firstly performed using module $\mathrm{S} 1$ to form $\mathbf{4 5}$. Subsequent treatment of $\mathbf{4 5}$ by module N1 (CjCgtA and UDP-GalNAc) formed the $\beta 1-3 \mathrm{Gal}$ branch in the fully assembled compound 46. Sequential glycosylations of 46 at the $\beta 1-6$ GlcNAc branch by enzyme modules G1, S1, and N1 yielded 47-49, which were previously identified in mammalian cells by CORA and $\beta$-elimination methods ${ }^{12,58}$. In addition, the $\beta 1-6 \mathrm{GlcNAc}$ branch of 45 was directly extended by modules G1, $\mathrm{S} 1$, and F2, respectively, to produce glycans 50-52. In parallel, core 2 structures with $\alpha 2-6$ sialylation at the $\beta 1-3 \mathrm{Gal}$ branch (compounds 53-59) were assembled in a similar sequential manner (Fig. 5). It is worth pointing out that the synthesis of compound 59 from 54 can also be achieved by Hm2FT-catalyzed a1,2 fucosylation (module F1) followed by Hp3FT-catalyzed a1,3fucosylation (module F2), as both fucosyltransferases have relatively relaxed acceptor specificities and can tolerate both non-fucosylated and mono-fucosylated acceptor substrates ${ }^{59}$. Non-sialylated core 2 O-glycans have also been found in mammalian cells. For example, compounds 60-62, 64, and 65 were previously identified from several cell lines including primary cells ${ }^{12}$. These structures were synthesized from 2 via a sequential modular assembly shown in Fig. 5. Specifically, the alpha-Gal epitope-presenting glycan 61 was obtained via direct a1-3 galactosylation of the $\beta 1-6$ GlcNAc branch in 60 by module G3. G3 contains bovine a1,3-galactosyltransferase (Ba3GalT) that can use both LacNAc and Lac disaccharides as acceptor substrates $^{48}$.

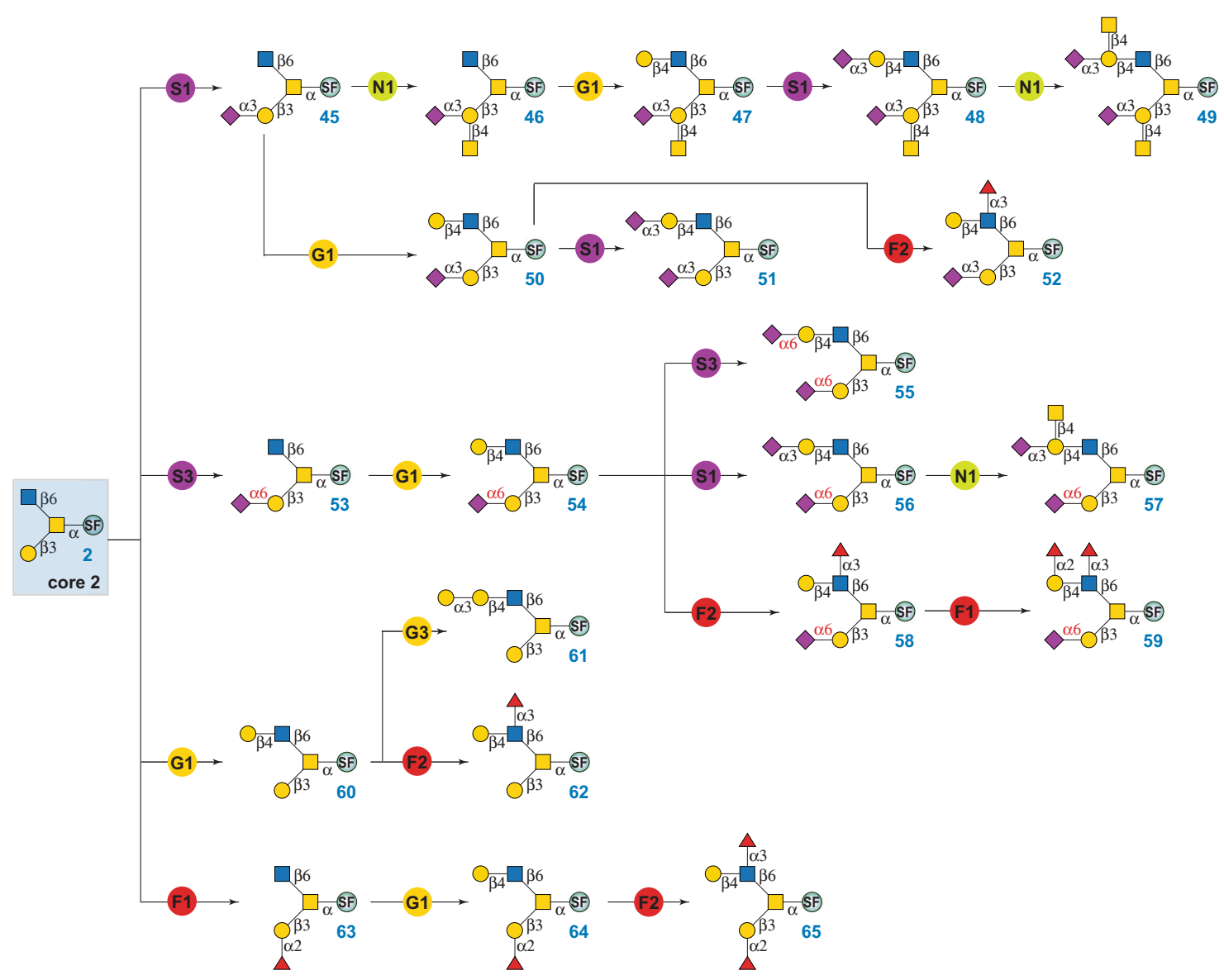

Fig. 5 Enzymatic modular synthesis of core 2 O-GalNAc glycans. G1: $\beta 1-4$ galactosylation with NmLgtB and UDP-Gal; G3: $\alpha 1-3$ galactosylation with B $\alpha 3$ GalT and UDP-Gal; S1: $\alpha 2-3$ sialylation with PmST1-M144D, NmCSS, CTP, and Neu5Ac; S3: $\alpha 2-6$ sialylation with Pd2,6ST, NmCSS, CTP, and Neu5Ac; N1: $\beta 1-4 N$-acetylgalactosaminylation with CjCgtA and UDP-GalNAc; F1: $\alpha 1-2$ fucosylation with Hm2FT and GDP-Fuc; F2: $\alpha 1-3$ fucosylation with Hp3FT and GDP-Fuc. SF, Fmoc protected Ser. 
Table 1 Ser-linked O-GaINAc glycans prepared in this study.

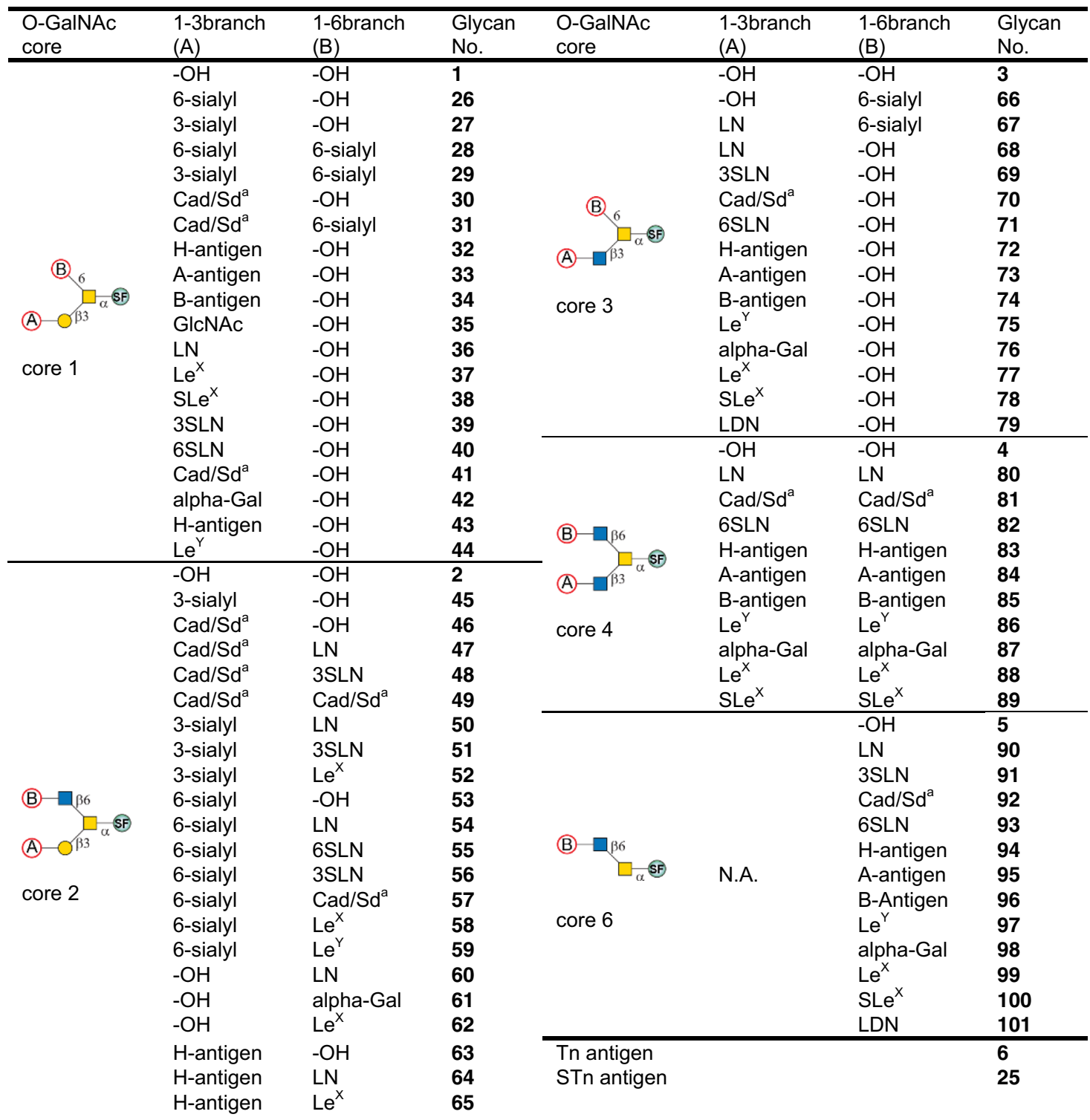

After validating the application of the CEMA strategy in the synthesis of cores 1 and 2 glycans, we turned our attention to synthesize low abundant O-GalNAc glycans. As shown in Supplementary Figures 1-3, fourteen core 3-type glycans (6679), ten core 4-type glycans (80-89), and twelve core 6-type glycans (90-101) were synthesized. Collectively, 83 Ser-linked OGalNAc glycans (Table 1, Supplementary Figure 4) were prepared through the robust CEMA strategy. All products were purified by RP-HPLC and characterized by NMR and mass spectrometry. Such glyco-amino acids can be applied, with or without protection by peracetylation, for solid-phase peptide synthesis ${ }^{60}$.

Microarray assays to probe the specificity of glycan-binding proteins (GBPs). Microarrays represent a major tool for functional glycomics studies. While various glycan microarrays have been developed during the last two decades, O-glycan structures in these arrays are still limited ${ }^{17,61}$. To explore the utility of the O-GalNAc-glycans synthesized, we prepared a synthetic glycan microarray according to the MIRAGE guidelines (Supplementary Table 1) by immobilizing Fmoc-deprotected glycans 1-6 and
25-101 on $\mathrm{N}$-hydroxysuccinimide (NHS)-activated glass slides. We assayed 17 commonly used GBPs and 2 recombinant influenza hemagglutinin (HA) proteins (Supplementary Table 2). The GBPs include fucose-binding lectins (AAL, UEA-I, LTL), sialic acid-binding lectins (MAL-I, SNA), LacNAc or GlcNAc-binding lectins (RCA-I, ECL, GSL-II, STL), Tn antigen-binding lectins (SBA, VVL, DBA), T antigen-binding lectins (PNA, Jacalin), and antibodies that are specifically against $\mathrm{sLe}^{\mathrm{X}}, \mathrm{STn}$, and $\mathrm{MUC1}$ (Fig. 6, Supplementary Figs. 5-10).

The glycan microarray results confirmed successful spotting of the glycans and revealed fine specificities of GBPs tested toward OGalNAc glycans. For example, the chitin-binding Solanum tuberosum lectin (STL) ${ }^{62}$ showed moderate binding to core 2 and 6 glycans presenting a terminal LacNAc motif (with/without further modifications) but not to core 1 and 3 structures (Fig. 6a), indicating its surprisingly strict preference toward the LacNAc on the $\beta 1-6 \mathrm{GlcNAc}$ branch. Such specificity may be applied to distinguish O-GalNAc core structures in heterogeneous mixtures. The $\mathrm{T}$ antigen-targeting Arachis hypogaea (peanut) lectin (PNA) and Jacalin were used as efficient tools for cancer diagnosis/prognosis and O-glycopeptide 

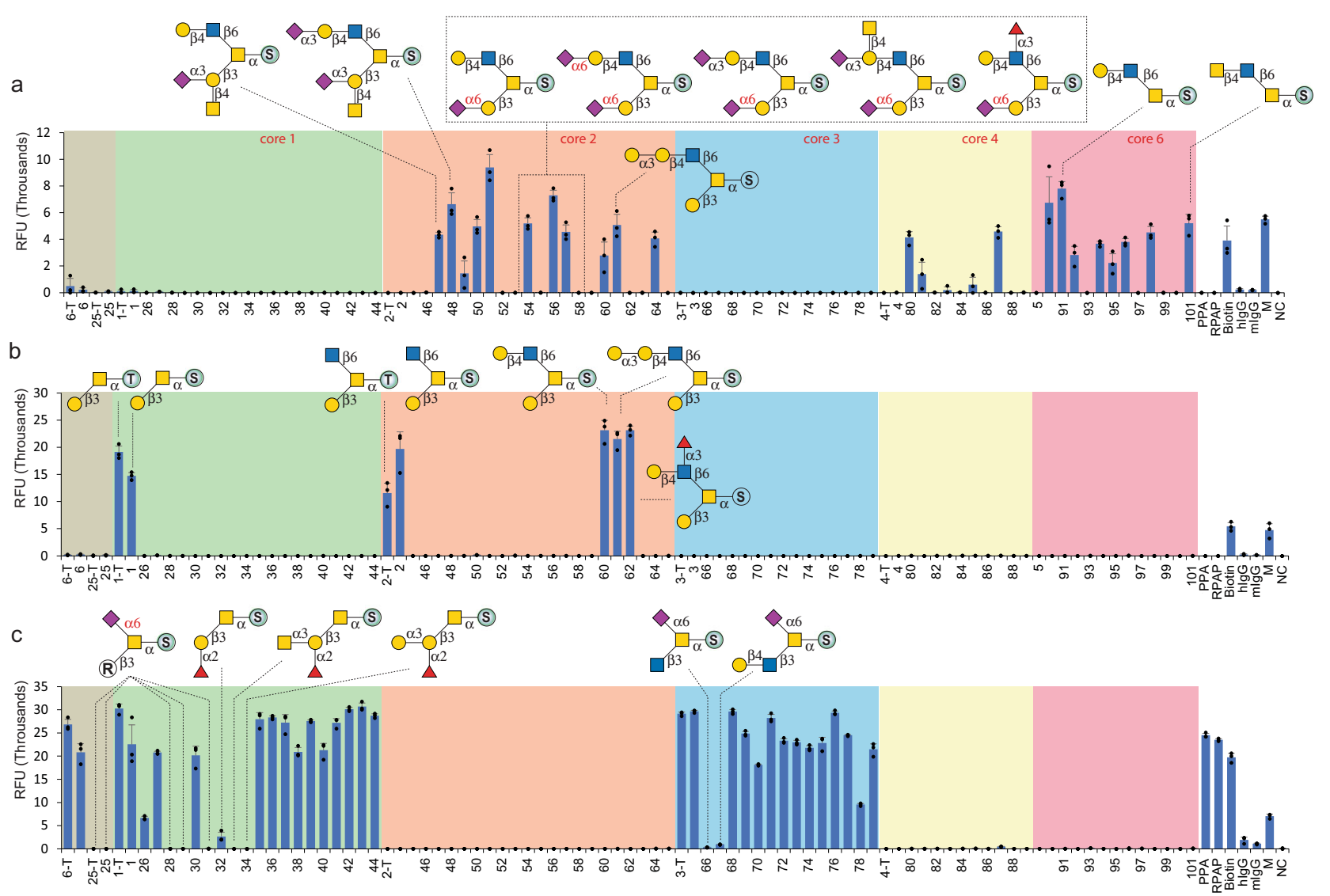

Fig. 6 Binding profiles of lectins towards O-GalNAc glycans. a The binding profile of STL towards O-GalNAc glycans. b The binding profile of PNA towards O-GalNAc glycans. c The binding profile of Jacalin towards O-GalNAc glycans. The $x$-axis shows glycans, and the $y$-axis shows relative fluorescence readout using Cy5-streptavidin $(1 \mu \mathrm{g} / \mathrm{mL})$. PPA = APGS(GalNAc $\alpha-)$ TAPP $(100 \mu M) ; \operatorname{RPAP}=\operatorname{TSAPD}(\mathrm{GalNAc} \alpha-)$ TRPAP $(100 \mu M) ; x-T$, Thr-linked counterparts of Ser-linked O-glycans; Biotin = biotinylated PEG amine $(0.01 \mathrm{mg} / \mathrm{mL})$; hlgG = human $\mathrm{lgG}(0.1 \mathrm{mg} / \mathrm{mL}), \mathrm{mlgG}=\mathrm{mouse} / \mathrm{gG}$ $(0.1 \mathrm{mg} / \mathrm{mL}) ; \mathrm{M}=$ Marker $(0.01 \mathrm{mg} / \mathrm{mL}$ Cy3-conjugated anti-Human IgG $+0.01 \mathrm{mg} / \mathrm{mL}$ Alexs647-conjugated anti-Human IgG); NC $=$ printing buffer negative control. $n=3$ independent replicates. The individual data points are shown as dots. Data are presented as mean values. Error bars represent standard deviation. Source data are provided as a Source Data file.

capturing ${ }^{63}$. We found that PNA selectively bound to T antigen (1) and core 2 glycans with an unmodified $\beta 1-3 \mathrm{Gal}$ branch $(\mathbf{2}, \mathbf{6 0}-\mathbf{6 2})$ (Fig. 6b), suggesting the requirement of a free Gal attached to the initiating GalNAc for proper recognition ${ }^{64}$. The results also showed that Jacalin bound strongly to all core 3 and nearly all core 1 glycans (devoid of a2-6 sialylation) at the same level of the Tn antigen, but did not bind to any core 2, 4, or 6 structures (Fig. 6c), suggesting a strict requirement for the free $\mathrm{C} 6-\mathrm{OH}$ on the initial GalNAc ${ }^{65}$. Binding specificities and fine details of all tested lectins are summarized in Supplementary Table 3. We then compared our lectin binding results with those obtained from the Consortium for Functional Glycomics (CFG) glycan microarray (Supplementary Table 4 summarizes O-GalNAc glycan structures in the array) (https://ncfg.hms.harvard.edu/ncfg-data/microarray-data/lectin-qua lity-assurancequality-control). The binding profiles of STL, PNA, VVL, SBA, and AAL to O-GalNAc glycans on the CFG array (Supplementary Figure 11) were similar to our results, further confirmed the successful fabrication of our synthetic O-GalNAcglycan array.

Altered expression levels of some serum anti-glycan antibodies have been linked to many diseases including cancer ${ }^{66-68}$, which are presumably the consequences of aberrant expression of the corresponding glycans including O-GalNAc glycans ${ }^{7-9}$. The synthetic O-GalNAc glycan microarray can be used to analyze the changes of anti-O-GalNAc glycan antibodies (IgG and IgM) in the sera of cancer patients and can serve as a promising platform for cancer biomarker discovery. To explore this opportunity, 29 serum samples from patients with colorectal cancer (Supplementary Table 5) and 29 from healthy controls were analyzed. The sera were diluted 50-fold and the antibodies bound to glycans on the array were probed with DyLight 650-conjugated antihuman IgG Fc antibody and DyLight 550-conjugated anti-human IgM antibody. For IgG, other than those bound strongly to $\mathrm{O}$ glycans presenting A- or B-antigen $(33,34,73,74,84,85,95,96)$ which are most likely the natural anti-A and anti-B antibodies, no apparent specific binding was observed (Supplementary Fig. 12). On the other hand, the overall IgM binding signals were high and varied significantly among individual serum samples (Fig. 7). Serum samples from both colorectal cancer patients and healthy controls showed higher overall IgM binding toward low abundant cores 3,4 , and 6 (compounds $3,4,5$ ) than to highly abundant cores 1 and 2 (compounds 1,2 ). The sera from patients with stage 3 colorectal cancer showed higher IgM binding than those from other patients, which was also observed previously by others in lung cancer ${ }^{18}$. However, no significant difference was observed between colorectal cancer samples and healthy controls. Interestingly, even though similar binding profiles to Ser-linked and Thr-linked O-glycans were observed for lectins (Fig. 6), the Ser or Thr amino acid residue in the O-GalNAc glycans made a significant difference in antibody binding. Stronger bindings to Ser-linked Tn (6), core 2 (2), core $3(3)$, and core 4 (4) than their Thr-linked counterparts (6-T, 2-T, 3-T, and 4-T) (Fig. 7, $p<0.01)$ 


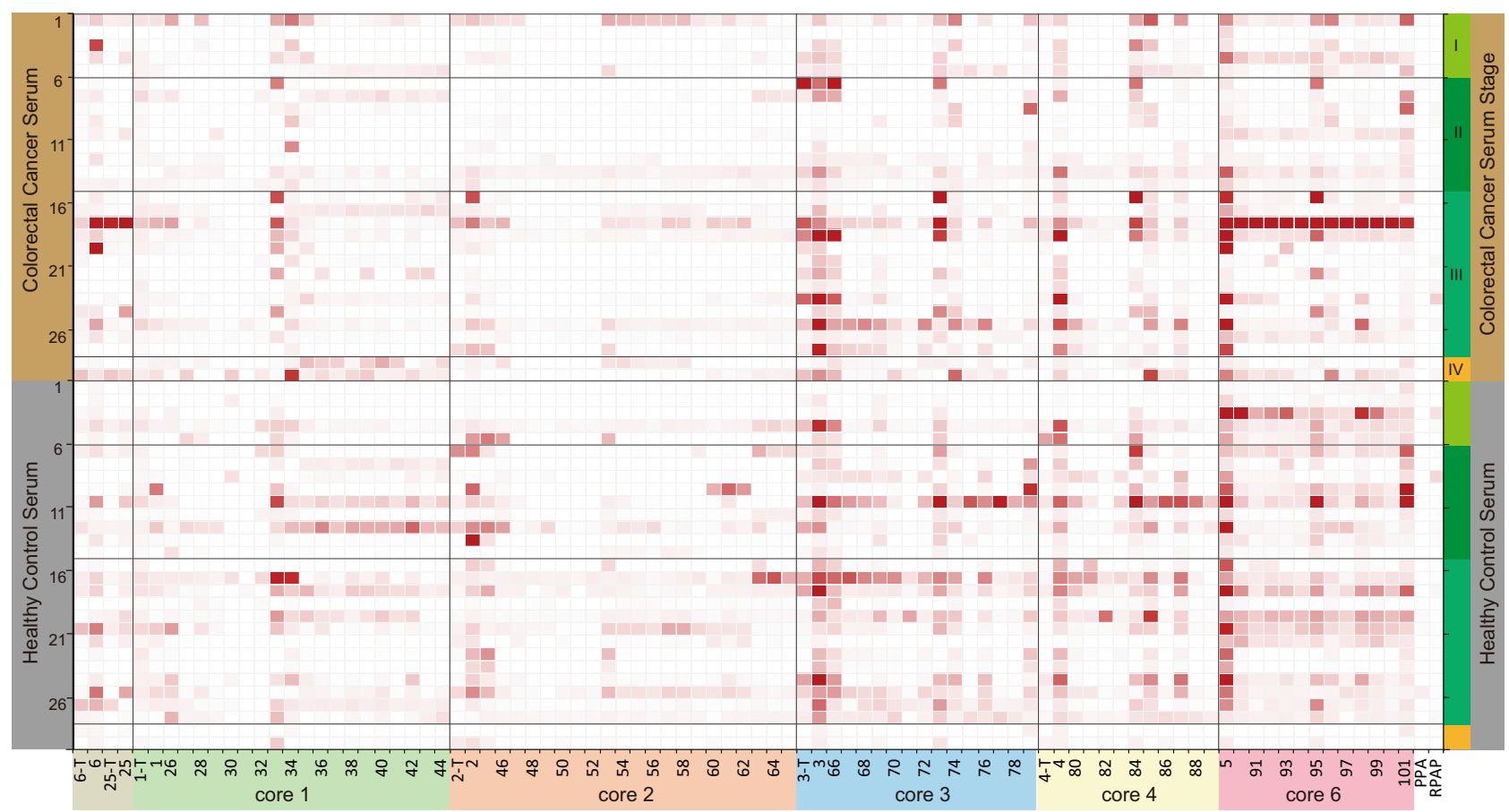

Fig. 7 Heatmap of IgM bindings on the $\mathbf{0}$-GalNAc glycan microarray in sera from colorectal cancer patients and healthy control people. PPA $=$ APGS $($ GalNAc $\alpha-)$ TAPP $(100 \mu M) ; R P A P=$ TSAPD $($ GalNAc $\alpha-)$ TRPAP $(100 \mu M) ; x-T$, Thr-linked counterparts of Ser-linked O-glycans; Biotin $=$ biotinylated PEG amine $(0.01 \mathrm{mg} / \mathrm{mL}) ; \mathrm{NC}=$ printing buffer negative control. $n=3$ independent replicates. Data are presented as mean values. Source data are provided as a Source Data file.

were observed for serum samples from both colorectal cancer patients and healthy controls. Moreover, glycan isomers presenting the same terminal epitopes, such as di-sialylated core 2 structures $(\mathbf{5 1}, \mathbf{5 5}$, and $\mathbf{5 6})$, showed a significant binding difference $(p<0.01)$ by IgM. A similar IgM binding difference was seen for glycan isomers $26 / 27$ and 69/71 $(p<0.01)$. The results suggested differential recognition of glycan isomers by serum anti-glycan antibodies. Another observation was that the extended O-glycans had lower overall IgM bindings than core structures (Fig. 7). Collectively, our results showed different serum antibody bindings to individual O-GalNAc cores as well as isomeric structures, providing insights to O-GalNAc glycan structure-activity relationship. Particularly, serum antibodies to rare O-GalNAc core 6 and less common cores 3 and 4 may serve as targets for cancer biomarker discovery.

In summary, we disclose an efficient chemoenzymatic modular assembly (CEMA) synthetic strategy that is used for the construction of a comprehensive cores 1-4 and 6-derived $\mathrm{O}$ GalNAc glycan library presenting numerous natural epitopes including LN, LDN, 3-sialyl, 6-sialyl, 3SLN, 6SLN, Le ${ }^{\mathrm{X}}$, SLe ${ }^{\mathrm{X}}, \mathrm{Le}^{\mathrm{Y}}$, and blood group $\mathrm{H}, \mathrm{A}, \mathrm{B}$, and $\mathrm{Cad} / \mathrm{Sd}^{\mathrm{a}}$ antigens. The CEMA strategy enables rapid access to 83 structurally diverse O-GalNAc glycans by deploying 3 chemical synthetic building blocks (a universal monosaccharide-amino acid acceptor 7 and two glycosyl donors $\mathbf{8}$ and 9) and 13 well-tailored glycosyltransferase modules in a precisely controlled sequential manner. The strategy can be readily adopted for the synthesis of cores 5, 7, and 8derived O-GalNAc glycans and other complex carbohydrates. The synthetic O-GalNAc glycan microarray represents a powerful platform to investigate the structure-function relationship of mucin O-glycans. In addition to being used directly in glycan-protein interaction studies, the structurally diverse, ready-to-conjugate glycan-amino acid probes can also be used as building blocks for solid-phase synthesis of glycopeptides.

\section{Methods}

General procedure of high-performance liquid chromatography. An analytical GL Science Inertsil ODS-4 column ( $100 \AA, 5 \mu \mathrm{m}, 4.6 \mathrm{~mm} \times 250 \mathrm{~mm})$ was used to monitor reactions and for final purity analysis. The signals were monitored by a UV detector $(260 \mathrm{~nm})$ or fluorescent detector (Ex $260 \mathrm{~nm}, \mathrm{Em} 310 \mathrm{~nm})$. Analysis was performed under a gradient running condition (solvent $\mathrm{A}: \mathrm{H}_{2} \mathrm{O}$ with $0.1 \%$ TFA; solvent B: acetonitrile with $0.1 \%$ TFA; flow rate: $1 \mathrm{~mL} / \mathrm{min}$; B\%: $20-40$ within $25 \mathrm{~min}$ ). With similar running conditions, the analytical Inertsil ODS-4 column was used for separating up to $3 \mathrm{mg}$ of products, and a semipreparative Inertsil ODS- 4 column $(100 \AA$, $5 \mu \mathrm{m}, 10 \mathrm{~mm} \times 250 \mathrm{~mm}$ ) was used for larger scales with a flow rate of $4 \mathrm{~mL} / \mathrm{min}$.

General procedure for enzymatic extensions. Reaction mixtures contain Tris$\mathrm{HCl}(100 \mathrm{mM}, \mathrm{pH} 7.5$ or 8.0$)$, an acceptor glycan $(10 \mathrm{mM})$, a donor $(15 \mathrm{mM})$, $\mathrm{MgCl}_{2}(10 \mathrm{mM})$, and an appropriate amount of enzyme. Reactions were incubated at $37^{\circ} \mathrm{C}$ and monitored by HPLC and/or MALDI-TOF MS. After over $90 \%$ acceptor was converted, the reaction was quenched, concentrated and subject to HPLC separation. Product-containing fractions were pooled and lyophilized for characterization and next step modular assembly.

Method for microarray fabrication. The O-GalNAc microarray was printed according to the guidelines of MIRAGE as summarized in Supplementary Table 1. Thr-linked O-glycans, O-glycopeptides PPA [APGS(GalNAca-)TAPP] and PPAP [TSAPD(GalNAca-)TRPAP] (Z Biotech), and Ser-linked O-glycans prepared in this study were prepared at a concentration of $100 \mu \mathrm{M}$ in the printing buffer $(150 \mathrm{mM}$ phosphate, $\mathrm{pH}$ 8.5), and printed on Nexterion slide H-3D hydrogel coated glass microarray slides (Applied Microarrays Inc), each for $400 \mathrm{pL}$ in triplicates. Printing buffer was printed as a negative control, biotinylated PEG amine $(0.01 \mathrm{mg} / \mathrm{mL})$, mouse IgG $(0.1 \mathrm{mg} / \mathrm{mL})$ and human $\operatorname{IgG}(0.1 \mathrm{mg} / \mathrm{mL})$ were printed in three replicates to serve as positive controls. A marker containing anti-human IgG-Cy3 conjugate $(0.01 \mathrm{mg} / \mathrm{mL})$ and anti-human IgG-Alexa647 conjugate $(0.01 \mathrm{mg} / \mathrm{mL})$ was printed in triplicates. A sciFLEXARRAYER S3 non-contacting ultralow volume dispensing system equipped with two PDC 80 Piezo dispense capillaries (Scienion) was used to spot glycans onto NHS-activated glass slides (16 subarrays on each slide). The spotting was carried out at room temperature with a humidity of $60 \%$, followed by overnight dehumidification. Printed slides were then soaked in blocking buffer ( $50 \mathrm{mM}$ ethanolamine, $100 \mathrm{mM}$ Tris- $\mathrm{HCl}, \mathrm{pH} 9.0$ ) for $2 \mathrm{~h}$, washed twice using MilliQ water, desiccated, and stored at $-20^{\circ} \mathrm{C}$ until use.

Method for microarray assay. All steps were performed at room temperature. Before assay, each subarray was separated by fitting the slides with ProPlate 16-well 
microarray modules and incubated using $100 \mu \mathrm{L}$ of TSMTB buffer $(20 \mathrm{mM}$ Tris- $\mathrm{HCl}$, $150 \mathrm{mM} \mathrm{NaCl}, 2 \mathrm{mM} \mathrm{CaCl}, 2$ mM MgCl $2,0.05 \%$ (v/v) Tween-20, 1\% (w/v) BSA, pH 7.4) for $10 \mathrm{~min}$. For assay, TSMTB was aspirated and $100 \mu \mathrm{L}$ of GBPs or serum samples at appropriate concentrations in TSMTB were added. The slides were then sealed and incubated for $1 \mathrm{~h}$ with gentle shaking, followed by washing for four times with TSMT buffer (TSMTB buffer without BSA). Next, $100 \mu \mathrm{L}$ of fluorescence-labeled secondary antibody or Cy5-streptavidin was added to each subarray, sealed, and incubated for $1 \mathrm{~h}$ with gentle shaking. After incubation, the slides were washed four times with TSMT, TSM (TSMT without Tween-20), and MilliQ water, respectively, and dried by brief centrifugation for scanning. A Genepix 4100A microarray scanner (Molecular Devices) was used to image the slides at $80 \%$ power and 500 or 600 PMT gains. The resultant images were analyzed using the Genepix Pro 6.1 software and processed using Excel to obtain microarray results. Biotin-labeled lectins were detected by Cy5-streptavidin $(1 \mu \mathrm{g} / \mathrm{mL})$. Anti-STn antibody, anti-MUC-1 antibody, anti-CD15 antibody $(10 \mu \mathrm{g} / \mathrm{mL})$ and anti-CD15s antibody $(10 \mu \mathrm{g} / \mathrm{mL})$ were detected by corresponding fluorescent-labeled secondary antibody $(5 \mu \mathrm{g} / \mathrm{mL})$. Recombinant influenza A virus hemagglutinin proteins were detected with Alexa 647-conjugated anti-His-tag antibody $(5 \mu \mathrm{g} / \mathrm{mL})$. Human serum specimens from colorectal cancer patients and healthy people were provided by Georgia Cancer Center at Augusta University and stored at $-80^{\circ} \mathrm{C}$ until use. The protocol for serum specimen preparation was approved by the Institutional Review Board of Augusta University and was performed in accordance with the Helsinki Declaration. All participants gave written informed consent. Human serum specimens were analyzed in a 1:50 dilution and detected using Dylight 650 anti-human IgG Fc (Invitrogen) and Dylight 550 antihuman IgM antibodies (Invitrogen) $(5 \mu \mathrm{g} / \mathrm{mL})$.

Reporting summary. Further information on research design is available in the Nature Research Reporting Summary linked to this article.

\section{Data availability}

The data supporting the findings of this study are available within the article and its Supplementary Information. Other relevant data are available from the corresponding author upon reasonable request. The Source data underlying Figs. 6, 7, Supplementary Figs. 5-11 are provided as a Source Data file. Source data are provided with this paper.

Received: 27 September 2020; Accepted: 29 April 2021;

Published online: 11 June 2021

\section{References}

1. Brockhausen I., Stanley P. O-GalNAc glycans. In Essentials of Glycobiology (ed^(eds rd, et al.) (2015).

2. Halim, A. et al. Site-specific characterization of threonine, serine, and tyrosine glycosylations of amyloid precursor protein/amyloid $\beta$-peptides in human cerebrospinal fluid. Proc. Natl Acad. Sci. USA 108, 11848-11853 (2011).

3. Steentoft, C. et al. Precision mapping of the human O-GalNAc glycoproteome through SimpleCell technology. EMBO J. 32, 1478-1488 (2013).

4. Linden, S. K., Sutton, P., Karlsson, N. G., Korolik, V. \& McGuckin, M. A. Mucins in the mucosal barrier to infection. Mucosal. Immunol. 1, 183-197 (2008).

5. Mayr, J. et al. Unravelling the role of O-glycans in influenza A virus infection. Sci. Rep. 8, 16382 (2018).

6. Carlin, A. F. et al. Molecular mimicry of host sialylated glycans allows a bacterial pathogen to engage neutrophil Siglec- 9 and dampen the innate immune response. Blood 113, 3333-3336 (2009).

7. Brockhausen, I. Mucin-type O-glycans in human colon and breast cancer: glycodynamics and functions. EMBO Rep. 7, 599-604 (2006).

8. Pinho, S. S. \& Reis, C. A. Glycosylation in cancer: mechanisms and clinical implications. Nat. Rev. Cancer 15, 540-555 (2015).

9. Burchell, J. M., Beatson, R., Graham, R., Taylor-Papadimitriou, J. \& TajaduraOrtega, V. O-linked mucin-type glycosylation in breast cancer. Biochem. Soc. Trans. 46, 779-788 (2018).

10. Kudelka, M. R., Ju, T., Heimburg-Molinaro, J. \& Cummings, R. D. Simple sugars to complex disease-mucin-type O-glycans in cancer. Adv. Cancer Res. 126, 53-135 (2015).

11. Cervoni, G. E., Cheng, J. J., Stackhouse, K. A., Heimburg-Molinaro, J. \& Cummings, R. D. O-glycan recognition and function in mice and human cancers. Biochem. J. 477, 1541-1564 (2020).

12. Kudelka, M. R. et al. Cellular O-glycome reporter/amplification to explore Oglycans of living cells. Nat. Methods 13, 81-86 (2016).

13. Jin, C. et al. Structural diversity of human gastric mucin glycans. Mol. Cell Proteom. 16, 743-758 (2017)

14. Song, X. et al. Shotgun glycomics: a microarray strategy for functional glycomics. Nat. Methods 8, 85-90 (2011).

15. Smith, D. F., Cummings, R. D. \& Song, X. History and future of shotgun glycomics. Biochem. Soc. Trans. 47, 1-11 (2019).
16. Song, X. et al. Oxidative release of natural glycans for functional glycomics. Nat. Methods 13, 528-534 (2016).

17. Li, Z. \& Chai, W. Mucin O-glycan microarrays. Curr. Opin. Struct. Biol. 56, 187-197 (2019).

18. Li, Z. et al. Amplification and preparation of cellular O-glycomes for functional glycomics. Anal. Chem. 92, 10390-10401 (2020).

19. Santra, A. et al. Regioselective one-pot multienzyme (OPME) chemoenzymatic strategies for systematic synthesis of sialyl core 2 glycans. ACS Catal. 9, 211-215 (2019).

20. Gutierrez Gallego, R. et al. Enzymatic synthesis of the core-2 sialyl Lewis X Oglycan on the tumor-associated MUCla' peptide. Biochimie 85, 275-286 (2003).

21. Dudziak, G. et al. In situ generated O-glycan core 1 structure as substrate for Gal(beta 1-3)GalNAc beta-1,6-GlcNAc transferase. Bioorg. Med. Chem. Lett. 8, 2595-2598 (1998).

22. Xia, J., Alderfer, J. L., Srikrishnan, T., Chandrasekaran, E. V. \& Matta, K. L. A convergent synthesis of core 2 branched sialylated and sulfated oligosaccharides. Bioorg. Med. Chem. 10, 3673-3684 (2002).

23. Sengupta, P., Misra, A. K., Suzuki, M., Fukuda, M. \& Hindsgaul, O. Chemoenzymatic synthesis of sialylated oligosaccharides for their evaluation in a polysialyltransferase assay. Tetrahedron Lett. 44, 6037-6042 (2003).

24. Peng, W. et al. Recent H3N2 viruses have evolved specificity for extended, branched human-type receptors, conferring potential for increased avidity. Cell Host Microbe 21, 23-34 (2017).

25. Nycholat, C. M. et al. Synthesis of biologically active N-and O-linked glycans with multisialylated poly-N-acetyllactosamine extensions using P. damsela alpha2-6 sialyltransferase. J. Am. Chem. Soc. 135, 18280-18283 (2013).

26. Pett, C. et al. Effective assignment of alpha2,3/alpha2,6-sialic acid isomers by LCMS/MS-based glycoproteomics. Angew. Chem. Int. Ed. 57, 9320-9324 (2018).

27. Pett, C. et al. Microarray analysis of antibodies induced with synthetic antitumor. Vaccines: Specificity against Diverse Mucin Core Structures. Chem. Eur. J. 23, 3875-3884 (2017).

28. Ohyabu, N. et al. An essential epitope of anti-MUC1 monoclonal antibody KL-6 revealed by focused glycopeptide library. J. Am. Chem. Soc. 131, 17102-17109 (2009).

29. Malaker, S. A. et al. The mucin-selective protease StcE enables molecular and functional analysis of human cancer-associated mucins. Proc. Natl Acad. Sci. USA 116, 7278-7287 (2019).

30. Cheng, H. et al. Synthesis and enzyme-specific activation of carbohydrate - geldanamycin conjugates with potent anticancer activity. J. Med. Chem. 48, 645-652 (2005).

31. Paulsen, H. \& Helpap, B. Building blocks of oligosaccharides. Part XCVI. Synthesis and partial structures of the N-glycoproteins of the complex type. Carbohydr. Res. 216, 289-313 (1991).

32. Wang, S. et al. Facile chemoenzymatic synthesis of O-mannosyl glycans. Angew. Chem. Int. Ed. 57, 9268-9273 (2018).

33. Marcaurelle, L. A. \& Bertozzi, C. R. Recent advances in the chemical synthesis of mucin-like glycoproteins. Glycobiology 12, 69R-77R (2002).

34. Cato, D., Buskas, T. \& Boons, G. J. Highly efficient stereospecific preparation of Tn and TF building blocks using thioglycosyl donors and the $\mathrm{Ph} 2 \mathrm{SO} / \mathrm{Tf} 2 \mathrm{O}$ promotor system. J. Carbohydr. Chem. 24, 503-516 (2005).

35. Ngoje, G., Addae, J., Kaur, H. \& Li, Z. Development of highly stereoselective GalN3 donors and their application in the chemical synthesis of precursors of Tn antigen. Org. Biomol. Chem. 9, 6825-6831 (2011).

36. Chamberland, S., Ziller, J. W. \& Woerpel, K. A. Structural evidence that alkoxy substituents adopt electronically preferred pseudoaxial orientations in sixmembered ring dioxocarbenium ions. J. Am. Chem. Soc. 127, 5322-5323 (2005).

37. Dinkelaar, J. et al. Stereodirecting effect of the pyranosyl C-5 substituent in glycosylation reactions. J. Org. Chem. 74, 4982-4991 (2009).

38. Kuduk, S. D. et al. Synthetic and immunological studies on clustered modes of mucin-related Tn and TF O-linked antigens: the preparation of a glycopeptide-based vaccine for clinical trials against prostate cancer. J. Am. Chem. Soc. 120, 12474-12485 (1998).

39. Shaik, A. A., Nishat, S. \& Andreana, P. R. Stereoselective synthesis of natural and non-natural thomsen-nouveau antigens and hydrazide derivatives. Org. Lett. 17, 2582-2585 (2015).

40. Crich, D. \& Sun, S. Are glycosyl triflates intermediates in the sulfoxide glycosylation method? A chemical and 1H, 13C, and 19F NMR spectroscopic investigation. J. Am. Chem. Soc. 119, 11217-11223 (1997).

41. Kalikanda, J. \& Li, Z. Study of the stereoselectivity of 2-azido-2deoxygalactosyl donors: remote protecting group effects and temperature dependency. J. Org. Chem. 76, 5207-5218 (2011).

42. Paulsen, H. \& Hölck, J.-P. Synthese der glycopeptide O- $\beta$-D-galactopyranosyl(1-3)-O-(2-acetamido-2-desoxy- $\alpha$-D-galactopyranosyl)-(1-3)-L-serin und-Lthreonin. Carbohydr. Res. 109, 89-107 (1982).

43. Santra, A., Ghosh, T. \& Misra, A. K. Expedient synthesis of two structurally close tetrasaccharides corresponding to the O-antigens of Escherichia coli O127 and Salmonella enterica O13. Tetrahedron: Asymmetry 23, 1385-1392 (2012) 
44. Das, R. \& Mukhopadhyay, B. Chemical O-glycosylations: an overview. Chem. Open 5, 401-433 (2016).

45. Knerr, P. J. \& van der Donk, W. A. Chemical synthesis and biological activity of analogues of the lantibiotic epilancin 15X. J. Am. Chem. Soc. 134, 7648-7651 (2012).

46. Adamo, R. \& Kováč, P. Glycosylation under thermodynamic control: synthesis of the di- and the hexasaccharide fragments of the O-SP of vibrio cholerae O:1 serotype Ogawa from fully functionalized building blocks. Eur. J. Org. Chem. 2007, 988-1000 (2007).

47. Lau, K. et al. Highly efficient chemoenzymatic synthesis of beta1-4-linked galactosides with promiscuous bacterial beta1-4-galactosyltransferases. Chem. Commun. 46, 6066-6068 (2010).

48. Fang, J. et al. Highly efficient chemoenzymatic synthesis of $\alpha$-galactosyl epitopes with a recombinant a (1-3)-galactosyltransferase. J. Am. Chem. Soc. 120, 6635-6638 (1998).

49. McArthur, J. B., Yu, H., Zeng, J. \& Chen, X. Converting Pasteurella multocidaalpha2-3-sialyltransferase 1 (PmST1) to a regioselective alpha2-6sialyltransferase by saturation mutagenesis and regioselective screening. Org. Biomol. Chem. 15, 1700-1709 (2017).

50. Yu, H. et al. Highly efficient chemoenzymatic synthesis of naturally occurring and non-natural alpha-2,6-linked sialosides: a P. damsela alpha-2,6sialyltransferase with extremely flexible donor-substrate specificity. Angew. Chem. Int. Ed. Engl. 45, 3938-3944 (2006).

51. Wen, L. et al. A one-step chemoenzymatic labeling strategy for probing sialylated Thomsen-Friedenreich antigen. ACS Cent. Sci. 4, 451-457 (2018).

52. Sugiarto, G. et al. A sialyltransferase mutant with decreased donor hydrolysis and reduced sialidase activities for directly sialylating lewisx. ACS Chem. Biol. 7, 1232-1240 (2012)

53. Meng, X. et al. Regioselective chemoenzymatic synthesis of ganglioside disialyl tetrasaccharide epitopes. J. Am. Chem. Soc. 136, 5205-5208 (2014).

54. Yu, H. et al. Sequential one-pot multienzyme chemoenzymatic synthesis of glycosphingolipid glycans. J. Org. Chem. 81, 10809-10824 (2016).

55. Liu, Y. et al. A general chemoenzymatic strategy for the synthesis of glycosphingolipids. Eur. J. Org. Chem. 2016, 4315-4320 (2016).

56. Yi, W., Shen, J., Zhou, G., Li, J. \& Wang, P. G. Bacterial homologue of human blood group A transferase. J. Am. Chem. Soc. 130, 14420-14421 (2008).

57. Ye, J. et al. Diversity-oriented enzymatic modular assembly of ABO histoblood group antigens. ACS Catal. 6, 8140-8144 (2016).

58. Kawar, Z. S., Johnson, T. K., Natunen, S., Lowe, J. B. \& Cummings, R. D PSGL-1 from the murine leukocytic cell line WEHI-3 is enriched for core 2based O-glycans with sialyl Lewis x antigen. Glycobiology 18, 441-446 (2008).

59. $\mathrm{Wu}, \mathrm{Z}$. et al. Identification of the binding roles of terminal and internal glycan epitopes using enzymatically synthesized $\mathrm{N}$-glycans containing tandem epitopes. Org. Biomol. Chem. 14, 11106-11116 (2016).

60. Guo, Z. \& Shao, N. Glycopeptide and glycoprotein synthesis involving unprotected carbohydrate building blocks. Med. Res. Rev. 25, 655-678 (2005).

61. Wang, L. et al. Cross-platform comparison of glycan microarray formats. Glycobiology 24, 507-517 (2014).

62. Itakura, Y., Nakamura-Tsuruta, S., Kominami, J., Tateno, H. \& Hirabayashi J. Sugar-binding profiles of chitin-binding lectins from the hevein family: a comprehensive study. Int. J. Mol. Sci. 18, (2017).

63. Poiroux, G., Barre, A., van Damme, E. J. M., Benoist, H. \& Rouge, P. Plant lectins targeting o-glycans at the cell surface as tools for cancer diagnosis, prognosis and therapy. Int. J. Mol. Sci. 18, (2017).

64. Chandrasekaran, E. V. et al. Novel interactions of complex carbohydrates with peanut (PNA), Ricinus communis (RCA-I), Sambucus nigra (SNA-I) and wheat germ (WGA) agglutinins as revealed by the binding specificities of these lectins towards mucin core-2 O-linked and $\mathrm{N}$-linked glycans and related structures. Glycoconj J. 33, 819-836 (2016).

65. Tachibana, K. et al. Elucidation of binding specificity of Jacalin toward Oglycosylated peptides: quantitative analysis by frontal affinity chromatography. Glycobiology 16, 46-53 (2006).

66. Jacob, F. et al. Serum antiglycan antibody detection of nonmucinous ovarian cancers by using a printed glycan array. Int. J. Cancer 130, 138-146 (2012).

67. Pochechueva, $\mathrm{T}$. et al. Naturally occurring anti-glycan antibodies binding to Globo H-expressing cells identify ovarian cancer patients. J. Ovarian Res. 10, 8 (2017).

68. Tikhonov, A. A. et al. Analysis of anti-glycan IgG and IgM antibodies in colorectal cancer. Bull Exp. Biol. Med. 166, 489-493 (2019).
69. Yu, H., Yu, H., Karpel, R. \& Chen, X. Chemoenzymatic synthesis of CMPsialic acid derivatives by a one-pot two-enzyme system: comparison of substrate flexibility of three microbial CMP-sialic acid synthetases. Bioorg Med. Chem. 12, 6427-6435 (2004).

70. Gilbert, M. et al. Biosynthesis of ganglioside mimics in Campylobacter jejuni OH4384. J. Biol. Chem. 275, 3896-3906 (2000).

71. Peng, W. et al. Helicobacter pylori beta1,3-N-acetylglucosaminyltransferase for versatile synthesis of type 1 and type 2 poly-LacNAcs on N-linked, Olinked and I-antigen glycans. Glycobiology 22, 1453-1464 (2012).

72. Ramakrishnan, B. \& Qasba, P. K. Structure-based design of beta 1,4galactosyltransferase I (beta $4 \mathrm{Gal}-\mathrm{T} 1$ ) with equally efficient $\mathrm{N}$ acetylgalactosaminyltransferase activity. J. Biol. Chem. 277, 20833-20839 (2002).

73. Lin, S. W., Yuan, T. M., Li, J. R. \& Lin, C. H. Carboxyl terminus of Helicobacter pylori alpha1,3-fucosyltransferase determines the structure and stability. Biochemistry 45, 8108-8116 (2006).

\section{Acknowledgements}

This work was supported by National Institutes of Health (U01GM116263, U54HL142019, and R44GM123820). S.W. was partially supported by Molecular Basis for Disease (MBD) Doctoral Fellowship at Georgia State University. We thank Z biotech LLC (Aurora, CO) for microarray fabrication, and Dr. Xiu-Feng Wan (University of Missouri) for kindly providing recombinant HA proteins of H3N2 (A/Brisb-ane/10/ 2007) and H1N1 (A/New York/18/2009) (acquired from BEI resources).

\section{Author contributions}

L.L, P.G.W., W.G., and P.L. conceived and designed the project. M.R.G. and V.S. performed chemical synthesis; C.C. and W.G. performed enzymatic synthesis; H.Z. and D.I performed mass spectrometry analysis; C.C. and S.W. performed HPLC analysis; S.W. and M.R.G analyzed NMR spectra; S.W. performed glycan microarray binding assays; X.C. provided sialyltransferase plasmids; R.B. and K.L. collected and prepared the human serum specimens; M.R.G., S.W., C.C., and L.L. wrote the manuscript; W.G., F.W., and X.C. revised the manuscript, which was edited and approved by all authors.

\section{Competing interests}

The authors declare no competing interests.

\section{Additional information}

Supplementary information The online version contains supplementary material available at https://doi.org/10.1038/s41467-021-23428-x.

Correspondence and requests for materials should be addressed to P.G.W., P.L., W.G. or L.L.

Peer review information Nature Communications thanks the anonymous reviewer(s) for their contribution to the peer review of this work.

Reprints and permission information is available at http://www.nature.com/reprints

Publisher's note Springer Nature remains neutral with regard to jurisdictional claims in published maps and institutional affiliations.

Open Access This article is licensed under a Creative Commons Attribution 4.0 International License, which permits use, sharing, adaptation, distribution and reproduction in any medium or format, as long as you give appropriate credit to the original author(s) and the source, provide a link to the Creative Commons license, and indicate if changes were made. The images or other third party material in this article are included in the article's Creative Commons license, unless indicated otherwise in a credit line to the material. If material is not included in the article's Creative Commons license and your intended use is not permitted by statutory regulation or exceeds the permitted use, you will need to obtain permission directly from the copyright holder. To view a copy of this license, visit http://creativecommons.org/ licenses/by/4.0/

(c) The Author(s) 2021 